\title{
Social Support and Spiritual Well-being Role in Anticipating Suicidal Ideation during Covid-19 Pandemic among Malaysian University Students
}

Aini Azeqa Ma'rof, Nur Izzatie Mat Razi, Haslinda Abdullah

To Link this Article: http://dx.doi.org/10.6007/IJARBSS/v11-i12/11894

DOI:10.6007/IJARBSS/v11-i12/11894

Received: 09 October 2021, Revised: 10 November 2021, Accepted: 28 November 2021

Published Online: 16 December 2021

In-Text Citation: (Ma'rof et al., 2021)

To Cite this Article: Ma'rof, A. A., Razi, N. I. M., \& Abdullah, H. (2021). Social Support and Spiritual Well-being Role in Anticipating Suicidal Ideation during Covid-19 Pandemic among Malaysian University Students. International Journal of Academic Research in Business and Social Sciences, 11(12), 1510-1523.

Copyright: (c) 2021 The Author(s)

Published by Human Resource Management Academic Research Society (www.hrmars.com)

This article is published under the Creative Commons Attribution (CC BY 4.0) license. Anyone may reproduce, distribute, translate and create derivative works of this article (for both commercial and non0-commercial purposes), subject to full attribution to the original publication and authors. The full terms of this license may be seen at: http://creativecommons.org/licences/by/4.0/legalcode

Vol. 11, No. 12, 2021, Pg. 1510 - 1523

Full Terms \& Conditions of access and use can be found at http://hrmars.com/index.php/pages/detail/publication-ethics 


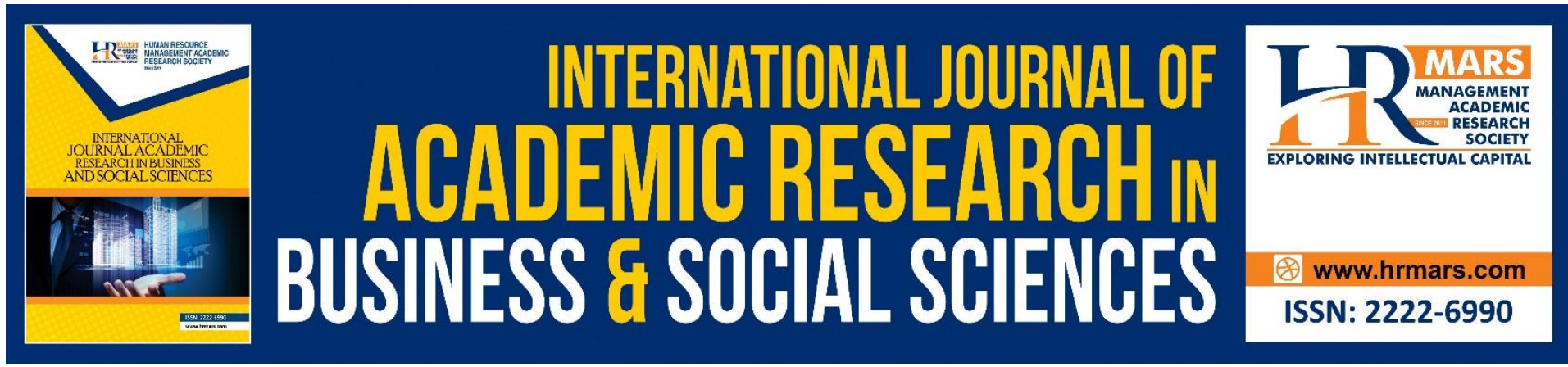

\title{
Social Support and Spiritual Well-being Role in Anticipating Suicidal Ideation during Covid-19 Pandemic among Malaysian University Students
}

\author{
Aini Azeqa Ma'rof, Nur Izzatie Mat Razi, Haslinda Abdullah \\ Faculty of Human Ecology, Universiti Putra Malaysia, 43400 Serdang, Selangor Darul Ehsan. \\ Email: azeqa@upm.edu.my
}

\begin{abstract}
Suicidal ideation is prevalent among teenagers and young adults in Malaysia as per reported by the Malaysia Ministry of Health. Meanwhile, there are significant concerns about the possibility of mental health problems occurring during Covid-19 pandemic. Communities have encountered mental health difficulties due to the morbidity, mortality, and mitigation actions associated with Covid-19. However, there has been dearth of empirical research on suicidal ideation during this uncertainty times especially the importance of the coping strategies applied. Thus, the purpose of this cross-sectional study is to examine the association between social support and spiritual well-being on suicidal ideation among young adults. A total of 407 young adults in Lembah Klang Valley participated in this study. The results revealed a significant negative correlation between spiritual well-being and all three types of social support: appraisal support, belonging support, and tangible support. Meanwhile, both suicidal ideation and spiritual well-being identified as significant predictors to suicidal ideation reduction with spiritual well-being as the strongest predictor. Thus, this study suggested that interventions that incorporate social support and spirituality may result in a more positive outcome in the fight against suicidal behavior in young adults. Study implications and recommendations were discussed.
\end{abstract}

Keywords: Suicidal Ideation, Social Support, Spiritual Well-being, Covid-19 Pandemic, Young Adults.

\section{Introduction}

The coronavirus disease pandemic (COVID-19) and its consequences have had a dramatic effect on healthcare systems, economy, and the health and well-being of people worldwide (McKee \& Stuckler, 2020). Additionally, the virus has sparked major concerns about the infection's possible impact on mental health. Public fear, sadness, worry, and tension have been described a result of concerns about infection, illness, and mortality (Reger et al., 2020). Moreover, the demand of social distancing and isolation during the pandemic aggravating stress and increasing vulnerability to mental disease (Torales et al., 2020). Meanwhile, numbers of research have found increase level of mental health concerns during the COVID19 pandemic (Gao et al., 2020; Zhu, et al., 2021). These elevated rates of mental health concerns had led to suicidal ideation and attempts (Hill et al., 2021). For instance, a recent 
study by Czeisler et al (2020) revealed that more than $40 \%$ of respondents reported experiencing negative mental health or increasing substance use that further led to suicidal ideation and self-harming. Additionally, study showed that those who were placed in shelterin-place or on lockdown during Covid-19 experienced an increase in suicide ideation over time compared to those who were not under the restrictions (Killgore et al., 2020).

Numerous studies have found various risk factors for suicidal thoughts, including major depression (Franklin et al., 2017), which could be exacerbated by the pandemic. According to Sahimi, et al (2021), majority of those having suicidal ideation and attempt for suicide during the pandemic reported suffered from the inability to bear the burden of heaps of responsibilities, employment issues, finances and family issues. Experts have speculated that social isolation, financial pressure, and mental health symptoms including stress and dread of Covid-19 itself could all contribute to an increase in suicide rates, dubbing the situation as a "perfect storm" (Reger et al., 2020). Notably, suicide was a significant cause of death, and the suicide rate was increasing even prior to the Covid-19 pandemic (Stone et al., 2018).

Suicidal ideation is the first step toward suicide. One of the primary reasons for the increase in suicide cases is an individual's proclivity for suicidal ideas or thoughts. A study conducted by the Malaysian Health Ministry $(\mathrm{MOH})$ under the National Health and Morbidity Survey (NHMS) 2017 has shown an increment in suicidal ideation and the number increased from $7.9 \%$ in 2012 to $10 \%$ in 2017 and reported to gradually increase to 20 percent by 2020 . Meanwhile, in 2019, the NHMS survey discovered that 424,000 children and young adults in Malaysia experienced mental health issues, with peer problems accounting for the majority (42.9 percent). Additionally, it is reported that approximately 500,000 adults, or $2.3 \%$ of the population suffered from depression. This is supported by 7 percent to 10 percent of individuals suffering from depression are estimated to be at risk of suicide in the next 10 to 15 years (Pan \& Arip, 2018).

Due to the recent hit of pandemic, empirical research on Covid-19 related suicidal ideation or thoughts and appropriate coping strategies is scarce. In the current study therefore sociopsychological factors in dealing with suicidal ideation was measured with a sample of Malaysian university students. It is said that coping techniques for stress influence the development of depression and suicidal ideation (Horwitz, Hill, \& King, 2011). Thus, it is crucial to establish the appropriate coping mechanisms in order to control and combat suicidal thoughts. Therefore, this study aimed at addressing social support and spiritual well-being as the crucial factors that would be linked with suicidal ideation or thoughts to be under control.

\section{Literature Review}

\section{Theoretical Explanation of Suicide Behavior}

Previous suicidal theories have focus on the development process and the cause of suicidal ideation. For instance, the Three Step Theory (3ST) by Klonsky and May (2015) hypothesized that suicidal thoughts is a result of a combination of pain, which is generally a psychological disorder and discomfort and there is a connection between the two major factors that result in an increase in suicidal ideas in an individual, to the point where the individual finally attempts suicide. Each attempt and effort taken by an individual to prevent suicide is preceded by suicidal thoughts. According to Joiner's (2005) Theory of Interpersonal Suicide Psychology, only those who desire and possess the potential to commit suicide are willing to 
try suicide. This notion is backed by suicide attempts undertaken by persons who have both suicidal thoughts and the acquired ability to commit suicide (Van Orden et al., 2010).

Additionally, Joiner (2005) contends that attempted suicide necessitates the acquisition of the ability to commit suicide, as a lethal suicide attempt entails high degrees of fear and pain. These individuals must possess a high level of ability and desire to deal with cases involving serious personal injury. According to the Theory of Interpersonal Suicide Psychology, an individual who is capable of committing suicide and has been subjected to provocative and painful situations that result in low levels of fear and a high tolerance for pain, such as trauma. According to Joiner (2005), there is a difference between individuals who have made several suicide attempts and those who have considered suicide but have not attempted to do it. Majority of individuals who struggle with this issue are not only willing to injure themselves but are also informed about the dangerous activity. Individuals who have the desire, ability, and motivation to injure themselves are at a high risk of engaging in serious suicidal behavior.

\section{Social Support as a Protective Factor against Suicide Risk}

Social support refers to the subjective availability of care and assistance received from social relationships. It is comprised of emotional support (e.g., expression of empathy), instrumental support (e.g., assistance with household tasks), and informational support (e.g., financial advice) from a variety of sources, including friends and family (House, Umberson, Landis, 1988). Findings from systematic reviews (Gariépy et al., 2016; Santini et al., 2015) and metaanalysis (Rueger et al., 2016) suggested that those who perceive more social support experience fewer depressive symptoms and those reduce the risk of suicidal ideation and attempts.

According to Lieberman et al (2005), perceived social support can be a predictor of suicidal ideation. This suggests that a high level of perceived social support can help prevent the emergence of suicidal ideation. This is consistent with Barrera's (2000) stress avoidance paradigm, which states that if an individual views their social support to be available and sufficient, they would be protected from negative impacts of life stressors. That is, if someone has a favorable view of social support, they will be more resilient prior to experiencing stress. Furthermore, support may also cause a reconsideration of a stressful situation, thereby inhibiting a maladaptive response. Cohen and Wills (1985) identify social resources that can act as stress buffers, including appraisal support (assistance in coping with stressful events), companionship or belongingness, and instrumental support (financial aid, material resources, services).

Social support is one of the potential resiliency factors that can control the level of suicidal thoughts (Kleiman \& Liu, 2014). A study by Kleiman and Liu (2013) revealed that individuals with a higher level of social support are approximately $30 \%$ less likely to attempt suicide in their lifetime than those with a lower level of social support, even after accounting for a variety of established risk and protective factors for suicidal thoughts. Study by Khazaeipour et al., (2017) perceived social support as the main factor reduces the likelihood of developing depression. Meanwhile, depression as the primary predictors of suicidal thoughts can be reduced with adequate perceived social support (Barrera, 2000; Cheung et al., 2006). 
Social support is critical during emerging adulthood (late adolescence to late twenties), a period marked by significant changes in social roles and responsibilities for instance living independently, establishing romantic relationships (Arnett et al., 2014) and an increased prevalence of mental health problems (Bruffaerts et al., 2019). Notably, mental health is a key risk factors contributing to suicidal behavior in young adults (Bilsen, 2018). It has been stated that suicides and overdoses case among young adults were already elevated even before pandemic started and it could be worse during this pandemic due to the outbreak because they mostly experienced in financial issues and psychologically stressed (Khazan, 2020).

It has been reported that during the outbreak, $78 \%$ of households with high school or college students report educational disruptions from COVID-19 and over 8 in 10 students experiencing these disruptions delineated increase in stress level. Almost $44 \%$ of students complain on worrying about the capability and performance to enroll or stay enrolled in college (Johnson, 2020). It was recorded that $87.7 \%$ number of students having mild, moderate, and severe anxiety whereas only $12.3 \%$ students recorded to have a normal anxiety state (Muhammad et al., 2020). Thus, in the present study, researcher will focus on the social support and spiritual well-being as factors in determination of suicidal ideation among public university students which is categorized within the age of young adults.

\section{Spiritual Well-Being and Suicidal Ideation}

When individuals face difficulties and hardships in life, such as the predominance of suicide ideation, it has been demonstrated that spiritual beliefs are beneficial (Francis \& Bance, 2017). Spirituality is more focused on individual practice and is typically associated with a sense of peace and purpose. According to Rovers and Kocum (2010), spiritual well-being is vital for human beings because it provides the required strength and motivation for stability, meaning, life satisfaction, and self-confidence. Spiritual well-being has two dimensions: a horizontal dimension (existential) that refers to a sense of purpose in life, as well as peace and contentment, and a vertical dimension (religious) that refers to a sense of well-being in a relationship with God or a higher power (Moberg, 2002; Omar \& Mutaz, 2017). Religion has always been associated with improved health habits, such as low smoking rates and decreased alcohol consumption, meanwhile spiritual intake helps regulate emotions, which has beneficial physiological effects. This is because all religions require their adherents to abstain from self-harming behavior.

Ibrahim et al., (2019) in a study on the relationship between spiritual well-being and suicide ideation among young adults showed that the higher spiritual well-being, the lower suicidal ideation. Meanwhile, according to Currier et al., (2018) indicated in a recent study that all severe spiritual components can be strongly associated to suicidal ideation. According to the past research, higher levels of spirituality may help buffer risk of active suicide desire and promote protective effect against depression (Souzan, Amal \& Samar, 2018). Without certainty, spiritual well-being is crucial in preventing suicide ideation. Thus, individuals with high spiritual intelligence may also be no exception in facing the tendency to create suicidal ideas due to the emotional burden they bear (Foo et al., 2019). However, there are researchers who find that traditional religiosity has little to do with lower levels of suicidal ideas (Robbins \& Francis, 2009). 
In a study conducted by Chen (2006) found that the combination of religious approach and social support can give a more positive impact on the self and emotional changes of an individual in the process of recovery. Meanwhile, perceived both high in social support and spiritual well-being can decrease the possibility of suicide ideation to emerge as it can act as a protective mechanism from negative effect of life stressor (Cho \& Haslam, 2010; Berra, 2000). Thus, considering a dearth of evidence regarding association between perceived social support and spiritual well-being on suicidal ideation, this study aimed to examine the relationship between these two factors on suicidal ideation among Malaysian university students.

\section{Method}

\section{Participants}

A total of 407 undergraduate public university students in Lembah Klang that comprise as young adults with majority of 255 (62.7\%) aged 21-25 years followed by 182 students within the age of $18-20$ years (37.3\%). 4 public universities in Lembah Klang were chosen. Majority of the students were female $(n=289,71 \%)$ and male $(n=128,29 \%)$.

\section{Data Collection}

A quantitative approach using a self-administered questionnaire was adopted by using a cross-sectional design method. The data was collected in May 2020 during the COVID-19 outbreak in Malaysia. University students were classified as undergraduates (within the young adults age range) from Malaysian public universities in Lembah Klang area. Three universities have been selected namely Universiti Kebangsaan Malaysia, Universiti Putra Malaysia and Universiti Malaya. The data collection involved online survey by using the Google Form where the link to the questionnaire has been shared around via online platform; WhatsApp and Telegram. Prerequisite for those from respective universities and within the age range was selected regardless of gender, religion, and marital status.

\section{Measures}

Social support. Social support was measured by using the Interpersonal Support Evaluation List - 12 (ISEL-12) by Cohen et al., (1985). This questionnaire consists of three dimensions of perceived social support: (1) appraisal, (2) belonging, and (3) tangible. There are 12 items in this questionnaire for instance, "If I wanted to go on a trip for a day (for example, to the country or mountains), I would have a hard time finding someone to go with me" (belonging subscale), "I feel that there is no one I can share my most private worries and fears with" (appraisal subscale), and "If I were sick, I could easily find someone to help me with my daily chores" (tangible subscale). Respondents were asked to rate to the statement on a four-point Likert scale ranging from 1 (definitely false) to 4 (definitely true). The reliability was $\alpha=.87$.

Spiritual well-being. Spiritual well-being was measured using the Spirituality Index of WellBeing (SIWB) scale developed by (Daeleman and Frey, 2004). This instrument measures respondent's perceptions of their spiritual quality of life by considering one's self-efficacy and life-schema with 12 items. Examples of the items are "There is not much I can do to help myself", "Often, there is no way I can complete what I have started", and "I can't begin to understand my problems". Respondents were asked to rate to the statement on a four-point likert scale ranging from 1 (strongly agree) to 4 (strongly disagree). The reliability was $\alpha=91$. 
Suicidal ideation. Suicidal ideation was measured using the Suicidal Ideation Attributes Scale (SIDAS) developed by (Van Spijker et al., 2014). This instrument is designed to screen individuals in the community for presence of suicidal thoughts and assess the severity of these thoughts. Examples of the items are "In the past month, how often have you had thoughts about suicide?" with a ten-point Likert scale ranging from 0 (Never), to 10 (Always), "In the past month, how much controls have you had over these thoughts? With a ten-point Likert scale ranging from 0 (No control), to 10 (Full control) and "In the past month, how close have you come to making a suicide attempt?" with a ten-point Likert scale ranging from 0 (Not close at all) to 10 (Made an attempt. The reliability was $\alpha=91$.

\section{Results and Discussion}

From Table 1, there is a negative significant relationship between social support on suicidal ideation $(r=-.34, p<.001)$ which indicates that the higher the social support, the lower the tendencies for the students to have a thought about suicide. This finding implies that students who are testified to have a higher score on perceived social support from their surrounding were more likely to have a lower score on suicidal ideation. This finding was supported by the past research stated that perceived social support can significantly predict the low level of suicide ideation (Nail \& Takwin, 2017). It is suggested that it is crucial to have someone on whom one can rely in times of need to provide moral and emotional support.

Moreover, the results indicate that the appraisal, tangible, and belonging ISEL scales were negatively correlated to suicidal ideation; appraisal support $(r=-.33, p<.001)$, belonging support $(r=-.27, p<.001)$, and tangible support $(r=-.35, p<.001)$. This result suggests that increasing in appraisal, tangible, and belongingness might directly reduce the for suicidal ideation. Therefore, this explains that these social supports elements can have a positive effect on increasing mental health wellness, and through this mechanism, act as a buffer to reduce risk indirectly.

Additionally, during the pandemic, it is crucial to identify the sort of social support in need by individual. For instance, tangible support which is based on a practical support such as material aid and behavioral assistance (Sherbourne \& Stewart, 1991) is important especially among students for them to survive during their online classes with proper equipment and tools. In addition, some students may need financial support especially for those stuck in university during the MCO where the university can assist in creating financial aid funds to those financially affected. Meanwhile, appraisal support where assistance given to those in need to cope with stressful events especially during this lockdown is also crucial. According to Johnson, Gooding, Wood et al (2010), positive self-appraisal may buffer the impact of hopelessness and thus reduce the suicidal ideation. Therefore, it is crucial for the institution to remain sensitive to students' mental health and to constantly promote the psychological support available at the university. Furthermore, for belonging support, less sense of belonging usually underlies by loneliness (Hatcher \& Stubbersfield, 2013). This study suggests that a sense of belongingness generate connectedness thus further may reduce the suicidal ideation. Throughout the MCO, social constraints force students to take online classes from home and engage in less physical engagement, particularly with their university friends. Additionally, students who are required or had to remain in university may have a greater proclivity for having less belonging support as a result of being stuck and trapped in their dormitories. However, even this study suggests that the students at this point having 
moderate belonging support, but it is crucial for the college fellow to always concern on the psychological well-being of the students.

Table 1: Correlation between Social Supports with Suicidal Ideation

\begin{tabular}{lcc}
\hline & Suicidal Ideation & \\
\hline & $\boldsymbol{r}$ & $\mathbf{p}$ \\
Social Support & $-.389^{* *}$ & .000 \\
Appraisal Support & $-.331^{* *}$ & .000 \\
Belonging Support & $-.286^{* *}$ & .000 \\
Tangible Support & $-.349^{* *}$ & .000 \\
Spiritual Well-Being & & .000 \\
\hline
\end{tabular}

Meanwhile, there was a negative significant correlation between spiritual well-being and suicidal ideation, $r=-.52, p<.001$. The finding illustrates that the higher the score on spiritual well-being among, the lower the score of suicidal ideations. This finding was supported by Norhayati et al. (2019) which found that spiritual well-being can be a protective factor of suicide ideation whereby young adults who are highly religious are more optimistic and believe that they can overcome obstacles, and, therefore, are less prone to suicidal behavior. From the context of this study, spiritual well-being focuses on the interpersonal spiritual wellbeing whereby looking into the self-efficacy and life schema instead from the religious aspect of spirituality. The result suggests that the student's belief in their capacity to execute behaviors necessary to produce specific performance attainments makes them resistant to suicidal risks.

Table 2: Multiple regression of social support and spiritual well-being on suicidal ideation

\begin{tabular}{|c|c|c|c|c|c|c|}
\hline Predictors & & B & SE & B & $\mathbf{t}$ & Sig. (p) \\
\hline Social Support & & 3.142 & 1.094 & .146 & 2.873 & .004 \\
\hline Spiritual Well-Being & & 5.351 & .631 & .430 & 8.474 & .000 \\
\hline $\mathrm{F}$ & 76.75 & & & & & \\
\hline$d f$ & 2 & & & & & \\
\hline $\mathrm{R}$ & .525 & & & & & \\
\hline$R^{2}$ & .275 & & & & & \\
\hline Adjusted $\mathrm{R}^{2}$ & .272 & & & & & \\
\hline
\end{tabular}

Meanwhile, multiple regression analysis was used to test if the social support or spiritual wellbeing were able to predict suicidal ideation significantly. Based on Table 2 , both social support $(b=.146, p=.004)$ and spiritual well-being $(b=-.430, p<.001)$ significantly contribute to the prediction of suicidal ideation among respondents with the predictors explained 28 percent of the variance. However, spiritual well-being showed the strongest proportion of variance in suicidal ideation, $F(2,404)=76.75, p<.001$. This result has been supported by previous study. Norhayati et al., (2019) showed that spiritual well-being contributed $8.7 \%$ of variance in suicide ideation. Meanwhile Foo et al., (2018) showed that both psychological and spiritual well-being can predict suicidal ideation. This study thus suggests that even social support that one's received from the surroundings or external is necessary, however, it is the internal factor such as spiritual well-being (i.e., self-efficacy and life schema) that operate as 
a buffer against suicidal ideation. The result further explains that those with strengthen spiritual well-being promotes resilience which can enhance competent functioning and life outcomes (Rutter, 2006).

\section{Further Discussion and Policy Implication}

From the study, result showed a statistically negative significant association between social support and suicidal ideation among respondents. This finding is consistent with previous studies that revealed high perceived social support strongly predict suicidal ideation (Norhayati et al., 2019; Menon et al., 2018). While social support is the general availability of friends and family members that provide psychological and material resources (Kleiman \& Riskind, 2012), this indicates that a lack of social support will increase the likelihood of developing suicidal ideation. According to Naila and Takwin (2018), perceived adequate social support is often a vital component of recovery from a variety of mental health problems, including suicidal ideation. This study also supported that social support may act as a buffer against suicidal thoughts. This can be explained by those who discerns social support, it may assist one's in changing the meaning of life stressors to a more positive alike, altering the circumstances, gaining control of the stressful situation, and altering one's reactions to the stressful scenario (Barrera, 2000).

Apart from that, social support provides a practical advantage that may be used in times of need, such as referring someone to a counselling centre or physically interfering with a suicide attempter. According to Orden, Witte, Cukrowicz et al., (2010), suicide attempters demonstrated multiple signs of poor social functioning including intentionally beginning a conflict with others and hostility in relationship that makes seeking for social support something out of necessity. Past research also revealed that individuals of any age who experience a severe lack of social support may develop a range of mental health problems, including the ability to commit suicide. Concerning with the current COVID-19 pandemic, majority of the respondents are at home staying with families due to the Movement Control Order (MCO). Even though it is said that social support can be directly access especially from family members and parents, somewhat this does not mean that they received the social support that they deserve. In fact, it adds to the stress that they already suffered from the erratic online classes, internet disruptions during online lectures, piles of assignments and not to mention the additional responsibilities of home tasks that makes it difficult for them to focus on their studies and likely ended up with severe stress, anxiety, and depression. Meanwhile, students living in university colleges (i.e., dormitories) experiencing the same emotional situation. Although there is no internet issue, many students reported severe stress and loneliness as a result of lack social support due to the MCO and stuck in their dormitories. Thus, by having appropriate social support, it may help in elevate one's mental well-being and less intention of suicidal.

Meanwhile, from the study, spiritual well-being not only reported having negative significant relationship with suicidal ideation, but it also even contributed as the strongest factor over social support in predicting suicidal ideation. This brings to a meaning that those with higher spiritual well-being may less likely having suicidal ideation. Spiritual well-being in terms of faith, religiosity, and meaning of life are important to students' life. As religion may offer a worldview about life and self to believers, young adults who are highly religious and spiritual are more optimistic and believe that they can overcome obstacles, and therefore, are less 
prone to suicidal behavior. For individual who sees the meaning and purpose of life, there is a sense of fulfilment, rationality, understanding of being and the search to lead to their real potential (Ramos et. al, 2019).

It could assert that suicide is a three-part act that incudes suicidal ideation, suicidal behavior, and attempted suicide. Suicidal ideation or thoughts, on the other hand, has been proven to be more prevalent than complete suicide. This assertion is borne out by a Malaysian Ministry of Health survey, which discovered that 6.3 percent of people aged 16-19 and 20-24 have the highest level of suicidal ideation $(\mathrm{MOH}, 2011)$. Suicidal ideation rates may be greater than reported because of the stigma associated with suicide and mental illness in developing Asian countries (Lauber \& Rossler, 2007). Additionally, the common sense of guilt perpetuates the culture of stigma around suicide (Kyle, 2013). Thus, this study is crucial to bring out in order to understand the suicidal scenario among the young adults particularly university students as well as the importance of social support and spiritual well-being and needs in assisting and empowering the students' mental health from deteriorating particularly during this COVID19 pandemic with many restrictions applied.

Suicide among young adults is a significant public mental health problem. Young adults are among the vulnerable category for mental health problems and its prevalence considerably climbs during the COVID-19 pandemic. The number of suicidal cases is increasing, and it remains the primary cause of mortality among young adults worldwide, accounting for significant number of premature deaths, as well as an enormous amount of unnecessary suffering and societal loss. Suicidal ideation is the consequence of a complex dynamic and unique interaction of several risk factors. On the other hand, the knowledge of risk factors is increasing substantially. Among the key risk factors include mental disorder, unique personality characteristics, genetic loading, and family processes, in combination with triggering psychosocial stressors, exposure to inspirational role models, and the availability of means of suicide.

As suicidal rate is increasing especially during the COVID-19 pandemic, the only way forward to combat this negative act is by providing support starting from whenever one's has the suicidal ideation. Thus, it is important to minimize the risk factors and maximize protective factors through integrated and multi-sector prevention initiatives. Instead of social support and spiritual well-being as supported by this study, other key prevention strategies can be applied such as population-based interventions, for instance, raising awareness through campaigns on mental resilience, careful media coverage, mental health promotion, and restricting access to means of suicide. Additionally, focus can bring to the high-risk subgroups by educating gatekeepers in different domains, providing crisis hotlines and online help, detecting and coaching dysfunctional families. Focus even can be made to individuals that identified as suicidal or having the suicide ideation by improving mental health treatment and providing strategies for coping with stress and grief (Calear et al., 2016). To increase the likelihood of future successful attempts to address young adults' suicide, further unravelling the complex suicide process must be accompanied by sustained and significant efforts scientifically underpin efforts and (re)evaluate existing and new prevention strategy plans, which is largely a matter of policy priorities and commitment. 


\section{Research Limitation and Future Research}

This study, however, has some limitations. With regards to the research design, the inability to establish causal relationships when all measures were obtained at the same time means that it was unable to ascertain the chronological order of the variables. As a result, it was unclear if it's a lack of social support or spiritual well-being preceded the development of suicidality. Moreover, this study focuses only to the university undergraduate students thus the findings may not be generalizable to other young adults from clinical populations. Addition to this, this study did not analyze the interaction between social support and spiritual well-being in order to determine whether the variables mediated or moderated each other. However, this is something to examine in further research especially by considering spiritual well-being as a mediator between the relations between social support and suicidal ideation.

Suggestions for future research, it would be beneficial to include different risk and protective factors to better understand the causes of suicide ideation among Malaysian young adults. Additionally, it is crucial to refine existing theoretical frameworks in connection to the role of social and psychological factors in suicide ideation in Malaysia, hence offering knowledge about the country's unique cultural context for suicide ideation.

\section{Conclusion}

Social support and spiritual well-being play a major role in predicting suicidal ideation. Interventions that incorporate social support and spirituality may result in a more favorable outcome for suicidal young adults especially beneficial to suicide prevention efforts. However, social support especially spirituality is a multidimensional concept and needs to be assessed accordingly. Thus, researchers that examine spirituality on mental health and suicidal context should acknowledge the sociodemographic, social, and health factors that are known to be risk factors for certain mental health problems and suicidal issues. Moreover, believing that one is isolated, unlovable, or incapable is associated with an increased risk of suicidal thoughts. Therefore, during the pandemic and $\mathrm{MCO}$, it is crucial for individual to look after their mental health, as well as the psychological well-being of others by providing the required assistance to avoid suicidal risks.

\section{Reference}

Adam, G., Horwitz, R. M. H., \& Cheryl, A. K. (2011). Specific coping behaviors in relation to adolescent depression and suicidal ideation. Journal of Adolescence, 34 (5), 10771085.

Arnett, J. J., Žukauskienė, R., \& Sugimura, K. (2014). The new life stage of emerging adulthood at ages 18-29 years: implications for mental health. Lancet Psychiatry, 1(7), 569-576.

Barrera, M. (2000). Social support research in community psychology. In Handbook of Community Psychology. New York: Kluwer Academic/Plenum Publishers, 215-245).

Bilsen, J. (2018). Suicide and Youth: risk factors. Frontiers in Psychiatry. 9, 540 - 545.

Bruffaerts, R., Mortier, P., Auerbach, R. P., Alonso, J., Hermosillo D., A. E., Cuijpers, P., Demyttenaere, K., Ebert, D. D., Green, J.G., Hasking, P., \& Stein, D. J. (2019). Lifetime and 12-month treatment for mental disorders and suicidal thoughts and behaviors among first year college students..International Journal of Methods Psychiatry Resources, 28(2). 
Calear, A. L., Christensen, H., Freeman, A., Fenton, K., Grant, J. B., Spijker, B. V., \& Donker, T. (2015). A systematic review of psychosocial suicide prevention interventions for youth. European Child and Adolescent Psychiatry, 25(5), 467-482.

Cheung, Law, C., Chan, B., Liu, \& Yip, P. (2006). Suicidal ideation and suicidal attempts in a population-based study of Chinese people: risk attributable to hopelessness, depression, and social factors. Journal of Affective Disorders, 90(3), 193-199.

Cohen, S., Mermelstein, R., Kamarck, T., \& Hoberman, H. M. (1985). Measuring the functional components of social support. In Social support: Theory, research and applications (pp. 73-94). Springer, Dordrecht.

Cohen, S., \& Wills, T. A. (1985). Stress, social support, and the buffering hypothesis. Psychological Bulletin, 98(2), 310-357.

Currier, J. M., Farnsworth, J. K., Drescher, K. D., McDermott, R. C., Sims, B. M., \& Albright, A. L. (2018). Development and evaluation of the expressions of moral injury scalemilitary version. Clinical Psychology and Psychotherapy, 25(3), 474-488.

Czeisler, M. É., Lane, R. I., Petrosky, E., Wiley, J. F., Christensen, A., Njai, R., Weaver, M. D., Robbins, R., Facer-Childs, E. R., Barger, L. K. Czeisler, C. A., \& Rajaratnam, S. M. (2020). Mental health, substance use, and suicidal ideation during the COVID-19 pandemicUnited States, June 24-30, 2020. Morbidity and Mortality Weekly Report, 69(32), 1049-1057.

Foo, S. J., Lo., K. W., Kamarul Z., W., Krishnan, S., \& Lokitashan, K. (2019). Psychological wellbeing and spiritual intelligence as predictors of suicidal ideation among emerging adults in Malaysia. International Journal of Education, Psychology and Counseling, 4, 139-148.

Francis, W., \& Bance, L. O. (2017). Protective role of spirituality from the perspective of Indian college students with suicidal ideation: "I am here because God exists". Journal of Religion and Health, 56(3), 962- 970.

Franklin, J. C., Ribeiro, J. D., Fox, K. R., Bentley, K. H., Kleiman, E. M., Huang, X., Musacchio, K. M., Jaroszewski, A. C., Chang, B. P., \& Nock, M. K. (2017). Risk factors for suicidal thoughts and behaviors: A meta-analysis of 50 years research. Psychology Bulletin, 143(2), 187-232.

Gao, J., Zheng, P., Jia, Y., Chen, H., Mao, Y., Chen, S., Wang, Y., Fu, H., \& Dai, J. (2020). Mental health problems and social media exposure during COVID-19 outbreak. PLoS One, 15(4).

Gariépy, G., Honkaniemi, H., \& Quesnel-Vallée, A. (2016). Social support and protection from depression: systematic review of current findings in Western countries. British Journal of Psychiatry, 209(4), 284-293.

Hatcher, S., \& Stubbersfield O. (2013). Sense of belonging and suicide: a systematic review. Canadian Journal of Psychiatry, 58(7), 432-6.

Hill, R. M., Rufino, K., Kurian, S., Saxena, J., Saxena, K., \& Williams, L. (2021). Suicide ideation and attempts in a pediatric emergency department before and during COVI-19. American Academic of Pediatrics, 147(3).

Horwitz, A. G., Hill, R. M., \& King, C. A. (2011). Specific coping behaviors in relation to adolescent depression and suicidal ideation. Journal of Adolescence, 34, 1077-1085.

House, J. S., Umberson, D., \& Landis, K. R. (1988). Structures and processes of social support. Annual review of sociology, 14(1), 293-318.

Ibrahim, N., Che Din, N., Ahmad, M., Amit, N., Ghazali, S. E., Wahab, S., Abdul Kadir, N. B., Halim, F. W. A., \& Halim, M. R. T. (2019). The role of social support and spiritual 
wellbeing in predicting suicidal ideation among marginalized adolescents in Malaysia. BMC Public Health, 19(553).

Institute for Public Health. (2011). National health and morbidity survey Kuala Lumpur: IPH. Ministry of Health Malaysia.

Johnson, J., Gooding, P. A., Wood, A. M., Taylor, P. J., Pratt, D., \& Tarrier, N. (2010). Resilience to suicidal ideation in psychosis: Positive self-appraisals buffer the impact of hopelessness. Behavior Research and Therapy Journal, 48(9), 883-889.

Johsnon, R. (2020). Students stressed out due to Coronavirus, new survey finds. https://www.bestcolleges.com/blog/coronavirus-survey/.

Joiner, Jr., T. E. (2005). Why people die by suicide. Cambridge, MA: Harvard University Press. Khazaeipour, Z., Hajiaghababaei, M., Mirminachi, B., Vaccaro, A. R., \& Rahimi-Movaghar, V. (2017). Social support and its association with depression, gender and socioeconomic indicators in individuals with spinal cord injury in Iran. Spinal Cord, 55(11), 1039-1044.

Khazan, O. (2020). “America's terrible internet is making quarantine worse. Why millions of students still can't get online". The Atlantic, https://www.theatlantic.com/technology/archive/2020/08/virtual-learning-whenyou-dont-have-internet/615322/.

Killgore, W. D. S., Cloonan, S. A., Taylor, E. C. Allbright, M. C., \& Dailey, N. S. (2020). Trends in suicidal ideation over the first three months of COVID-19 lockdowns. Psychiatry Research Journal, 293, 113390.

Kleiman, E. M., \& Riskind, J. H. (2012). Cognitive vulnerability to comorbidity: Looming cognitive style and depressive cognitive style as synergistic predictors of anxiety and depression symptoms. Journal of Behavior Therapy and Experimental Psychiatry, 43(4), 1109-1114.

Kleiman, E., M., \& Liu, R. T. (2013). Social support as a protective factor in suicide: Findings from two nationally representative samples. Journal of Affective Disorder, 150(2), 540545.

Klonsky, E. D., \& May, A. M. (2015). The three-step theory (3ST): A new theory of suicide rooted in the "ideation-to-action" framework. International Journal of Cognitive Therapy, 8(2), 114-129.

Kyle, J. (2013). Spirituality: its role as a mediating protective factor in youth risk for suicide. Journal of Spirituality in Mental Health, 15(10), 47-67.

Lauber, C., \& Rossler, W. (2007). Stigma towards people with mental illness in developing countries in Asia. International Review of Psychiatry, 19(2), 157-178.

Lieberman, R., Poland, S., \& Kornfeld, C. (2005). Best practices in suicide prevention and intervention in Best Practices in School Psychology, 273-288.

McKee, M., \& Stuckler, D. (2020). If the world fails to protect the economy, COVID-19 will damage health not just now but also in the future. Nature Medicine, 26, 640-642.

Menon, V., Subramaniam, K., Selvakumar, N., \& Kattimani, S. (2019). Suicide prevention strategies: An overview of current evidence and best practice elements.

Moberg, D. O. (2002). Assessing and measuring spiritualty: Confronting dilemmas of universal and particular evaluative criteris. Journal of Adult Development, 9(1), 47-60.

Muhammad, I., Faizah, S., Hooper, V. J., Akram., Q., \& Rosmaiza, A. G. (2020). The psychological impact of coronavirus on university students and its socio-economic determinants in Malaysia.

Naila, S., \& Takwin, B. (2018). Perceived Social Support as predictor of suicide ideation in Gunung Kidul High School students. 
Omar, I. A., \& Mutaz, F. A. (2017). Spiritual well-being perceived social support, and life satisfaction among university students. International Journal of Adolescence and Youth, 23(3), 1-8.

Orden, K. A. V., Witte, T. K., Cukrowicz, K. C., Braithwaite, S., Selby, E. A., \& Joiner, T. E. (2010). The interpersonal theory of suicide. Psychology Review, 117(2), 575-600.

Pan., H. K., \& Arip., M. A. S. M. (2018). Constructing validity and reliability of suicide tendency inventory. International Journal of Academic Research in Business and Social Sciences, 8(2), 467-481.

Ramos, M. R., Bennett, M. R., Massey, D. S., \& Hewstone, M. (2019). Humans adapt to social diversity over time. Proceedings of the National Academy of Sciences of the United States of America, 116(25), 12244-12249.

Reger, M. A., Stanley, I. H., \& Joiner, T. E. (2020). Suicide mortality and coronavirus disease 2019 - A perfect Storm. JAMA Psychiatry, 77(11), 1093-1094.

Rovers, M., \& Kocum, L. (2010). Development of a holistic model of spirituality. Journal of Spirituality in Mental Health, 12(1), 2-24.

Rueger, S. Y., Malecki, C. K., Pyun, Y., Aycock, C., \& Coyle, S. (2016). A meta-analytic review of the association between perceived social support and depression in childhood and adolescence. Psychology Bulletin. 142(10), 1017-1067.

Rutter, M. (2006). The Promotion of Resilience in the Face of Adversity. In A. Clarke-Stewart \& J. Dunn (Eds.), Families count: Effects on child and adolescent development (pp. 2652). Cambridge University Press.

Sahimi, H. M. S., Daud, T. I. M., Chan., L. F., Shah, S. A., Abd Rahman, F. H., \& Nik Jaafar, N. R. (2021). Depression and suicidal ideation in a sample of Malaysian Healthcare Workers: A preliminary study during the COVID-19 pandemic. Frontier Psychiatry.

Santini, Z. I., Koyanagi, A., Tyrovolas, S., Mason, C., \& Haro, J. M. (2015). The association between social relationships and depression: a systematic review. Journal of Affective Disorder, 175, 53-65.

Sherbourne, C. D., \& Stewart, A. L. (1991). The MOS social support survey. Social Science and Medicine, 32(6), 705-714.

Torales, J., O'Higgins, M., Castaldelli, M. J. M., \& Ventriglio, A. (2020). The outbreak of COVID19 coronavirus and its impact on global mental health. International Journal of Social Psychiatry, 66(4), 317-320.

Zhu, S., Wu, Y., Zhu, C. Y., Hong, W.C., Yu, Z. X., Chen, Z. K., Chen, Z. L., Jiang, D. G., \& Wang, Y. G. (2020). The immediate mental health impacts of the COVID-19 pandemic among people with or without quarantine managements. Brain Behavior and Immunity Journal, 87, 56-58. 